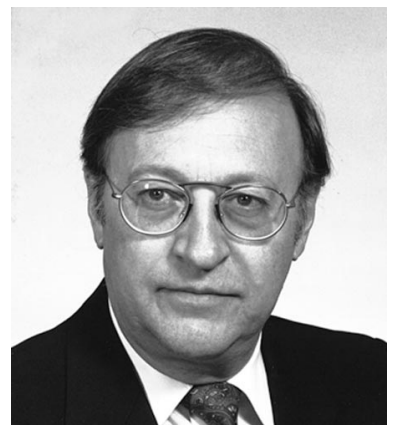

\section{Education and Training in Optics and Photonics}

The need for technicians, engineers, and scientists in the field of optical science and engineering is very important, and many countries perceive a challenge in trying to fill the gap between the existing brain and man power and the brain and man power required by the development of new industries in this domain. It is fantastic for those who have fought for the development of optics for many years to see their dreams becoming a reality. However, it is scary to see how many skilled people this new industry is looking for. It is especially scary when we see that the number of youths choosing a scientific career is not really increasing. We hope that the ETOP conferences will help in this way.

ETOP, which stands for Education and Training in Optics and Photonics, is a series of conferences run by the International Commission of Optics, SPIE-The International Society for Optical Engineering, and the Optical Society of America. These conferences are growing in importance since all developed countries have a big lack of the brain and man power required to fulfill the needs of industry.

Many groups, in many countries, think that the best way to start recruiting people for optical science and engineering must necessarily begin at the elementary school level. So, as did the SPIE Education Committee, those groups want to develop simple experiments and demonstrations that will generate interest and curiosity that children will surely want to satisfy. Unfortunately, the mission that the SPIE Education Committee gave to itself is very well adapted for U.S. education but not as well adapted for other countries. It should be very important to include in this committee volunteers from different ethnic communities since perception and understanding is largely dependent on each individual culture. It would be very interesting to put together all that expertise to look for a common denominator that would permit identification of the best approach in each culture. The same could be done at the high school level, since the passage from elementary schools to technical schools or universities varies a lot from one country to another, again depending on the cultural background. Consequently, working on a $\mathrm{K}-12$ education kit may give tools to educators in many countries, but in some countries, they will not know how to really adapt it for their needs.

I had the chance in the last nine months to participate in the ETOP conferences in Mexico, Singapore, and Senegal. In each of those conferences, it is evident that the difference between Quebec, Canada, France, Mexico, Senegal, and the U.S. is so large that although we can find some very promising candidates for the booming industry in optical science and engineering, we are all working with such unequal tools that it makes exchange difficult. In America, we are discussing ways to attract youths to optical science and engineering when not all fields of science and engineering include optics. However, I saw in Senegal, for example, that their needs are basic papers and basic apparatus to do experimental work to train their students. High-speed communication is not yet there even though they use cellular phones everywhere.

My recent experience in Senegal, talking with scientists from surrounding countries, shows me that since we cannot fulfill here the needs expressed by our rapidly developing industry, we must be prepared to spend time and funds to help those countries that do their best with what they have, even though their economy does not permit them to buy equipment. We can find in some countries very good scientists who will do theoretical work, but it is out of their reach to do experimental work because they cannot afford to buy experimental equipment.

I will not go farther on this topic since I would like to keep this editorial short, but I hope that these few lines will say just enough to you that you will start searching for ways to help those scientists who really want to join the optical science and engineering club but from which they all will be excluded if we are not doing our best to help them. I think this can and must be done for the benefit of our field.

Roger A. Lessard Editor 